\title{
On the equivalence between magnetic-field-induced phase transitions in the integer quantum Hall effect
}

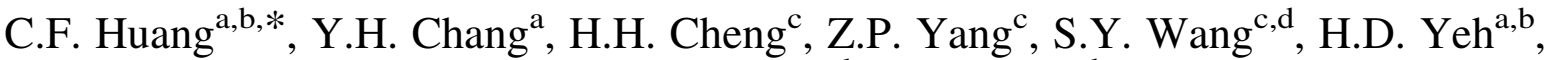 \\ H.T. Chou ${ }^{\mathrm{a}}$, C.P. Lee ${ }^{\mathrm{d}}$, G.J. Hwang \\ ${ }^{a}$ Department of Physics, National Taiwan University, Taipei 106, Taiwan, ROC \\ ${ }^{\mathrm{b}}$ National Measurement Laboratory, Center for Measurement Standards, Industrial Technology Research Institute, Bldg 16, \\ 321 Kuang Fu Road, Section 2, Hsinchu 300, Taiwan, ROC \\ ${ }^{\mathrm{c}}$ Center for Condensed Matter Sciences, National Taiwan University, Taipei 106, Taiwan, ROC \\ ${ }^{\mathrm{d}}$ Department of Electronics Engineering, National Chiao Tung University, Hsinchu 300, Taiwan, ROC
}

Received 16 December 2002; accepted 30 January 2003 by A.H. MacDonald

\begin{abstract}
Magnetic-field-induced phase transitions in the two-dimensional electron system in a AlGaAs/InGaAs/GaAs heterostructure are studied. Two kinds of magnetic-field-induced phase transitions, plateau-plateau $(\mathrm{P}-\mathrm{P})$ and insulator-quantum Hall conductor $(\mathrm{I}-\mathrm{QH})$ transitions, are observed in the integer quantum Hall effect regime at high magnetic fields. In the $\mathrm{P}-\mathrm{P}$ transition, both the semicircle law and the universality of critical conductivities are broken and we do not observe the universal scaling. However, the $\mathrm{P}-\mathrm{P}$ transition can still be mapped to the $\mathrm{I}-\mathrm{QH}$ transition by the Landau-level addition transformation, and as the temperature decreases the critical points of these two transitions appear at the same temperature. Our observations indicate that the equivalence between $\mathrm{P}-\mathrm{P}$ and $\mathrm{I}-\mathrm{QH}$ transitions can be found by the suitable analysis even when some expected universal properties are invalid.
\end{abstract}

(C) 2003 Elsevier Science Ltd. All rights reserved.

PACS: $73.40 \mathrm{Hm}$

Keywords: D. Phase transitions; D. Quantum Hall effect; D. Scaling

Magnetic-field-induced phase transitions observed in a twodimensional electron system (2DES) have attracted much attention [1-15]. Applying a magnetic field $B$ perpendicular to a two-dimensional system, in the quantum Hall $(\mathrm{QH})$ regime we can observe two types of magnetic-field-induced phase transitions, the plateau-plateau $(\mathrm{P}-\mathrm{P})$ and insulatorquantum Hall conductor $(\mathrm{I}-\mathrm{QH})$ transitions [1-3]. The former is observed between two quantum Hall states while the latter is observed between the insulating and quantum

\footnotetext{
* Corresponding author. Address: National Measurement Laboratory, Center for Measurement Standards, Industrial Technology Research Institute, Bldg 16, 321 Kuang Fu Road, Section 2, Hsinchu 300, Taiwan, ROC. Tel.: +886-357-321-01; fax: + 886-357-24-952.

E-mail address: d86222006@ms86.ntu.edu.tw (C.F. Huang).
}

Hall states. These two types of magnetic-field-induced phase transitions, in fact, are expected to be of the same universal class $[1,2]$. All the magnetic-field-induced phase transitions should follow the scaling with the universal critical exponent $\kappa=0.42 \pm 0.04$ [4,5] and obey the semicircle law [6-8]. According to the scaling, every magnetic-field-induced phase transition shrinks to a critical point as the temperature $T$ approaches zero $[1,4,8]$. The universality of the critical conductivities, i.e. the longitudinal and Hall conductivities $\sigma_{x x}$ and $\sigma_{x y}$ at the critical points, is also expected [2,9]. In the integer quantum Hall effect (IQHE), both $\mathrm{P}-\mathrm{P}$ and $\mathrm{I}-\mathrm{QH}$ transitions occur as the Fermi energy passes through the extended states of a Landau band as $B$ changes [1,2]. It was shown that in the IQHE, we can map the $\mathrm{P}-\mathrm{P}$ transition to the $\mathrm{I}-\mathrm{QH}$ transition by 
performing the Landau-level addition transformation to subtract the contributions of the Landau bands in which all states are below the Fermi energy and are filled $[1,2,8]$.

It was shown by Shahar et al. [2] that $\mathrm{P}-\mathrm{P}$ and $\mathrm{I}-\mathrm{QH}$ transitions are equivalent, and we can see the equivalence by examining the semicircle law, reflection symmetry, universal scaling, and the universality of critical conductivities. However, while the universality of $\kappa$ is expected and can be taken as a quantitative evidence for the equivalence between magnetic-field-induced phase transitions $[1,5,14,15]$, there are reports on the deviation of the critical exponent $\kappa[10$, 11]. In fact, Shahar et al. [12] later showed that magneticfield-induced phase transitions may not have the expected scaling behaviors. The validity of the semicircle law and the universality of critical conductivities are also questionable $[8,13,16]$. These unexpected results could be due to the inhomogeneity [14], mixing between Landau bands [16,17], small spin-splitting [8], or finite temperatures [14,15]. In the high-mobility two-dimensional systems, we also need to take into account the nonlocal effects due to the suppression of interchannel scattering between edge and bulk currentcarrying channels [18]. Actually the effective strength of the disorder potential experienced by a two-dimensional system can change with the sweeping magnetic field [1]. At low $B$, it was shown that the merge of extended states of different Landau bands could induce $\mathrm{I}-\mathrm{QH}$ transitions which are inconsistent with the global phase diagram (GPD) of the quantum Hall effect [19-22]. In fact, most realistic twodimensional systems at low $B$ reveal little about the magnetic-field-induced phase transitions because the localization length can be much larger than the sample sizes and the inelastic scattering length $[1,23]$. Recently, there are reports on the revival of the universality [24] and on how to subtract inhomogeneity effects near the critical points [14].

To further examine the equivalence between magneticfield-induced phase transitions in the IQHE, we report the study on the IQHE of the $2 \mathrm{DES}$ in a $\mathrm{AlGaAs} / \mathrm{InGaAs} / \mathrm{GaAs}$ heterostructure. At high fields, we observed the $\mathrm{P}-\mathrm{P}$ transition between $\nu=1$ and 2 quantum Hall states and the $\mathrm{I}-\mathrm{QH}$ transition from $\nu=1$ quantum Hall state to the insulating state, where $\nu$ presents the filling factor. For convenience, we denote the observed $\mathrm{P}-\mathrm{P}$ and $\mathrm{I}-\mathrm{QH}$ transitions as the $1-2$ and $0-1$ transitions. The number ' 0 ' denotes the insulating state while the numbers ' 1 ' and ' 2 ' denote the $\nu=1$ and 2 quantum Hall states, respectively [1, 2]. In our study, expected properties such as the semicircle law, the universal scaling, and the universality of critical conductivities are not observed in the 1-2 transition. By suitable analysis, however, we can still find evidences to support the equivalence between magnetic-field-induced phase transitions in the IQHE.

The sample used for this study is an AlGaAs/InGaAs/GaAs heterostructure. Fig. 1 shows its structure, in which the $2 \mathrm{DES}$ is located in the $\mathrm{In}_{0.15} \mathrm{Ga}_{0.85}$ As quantum well. The Hall pattern was made by the standard lithography and etching processes, and the $\mathrm{Au} / \mathrm{Ge}$ alloy was alloyed into

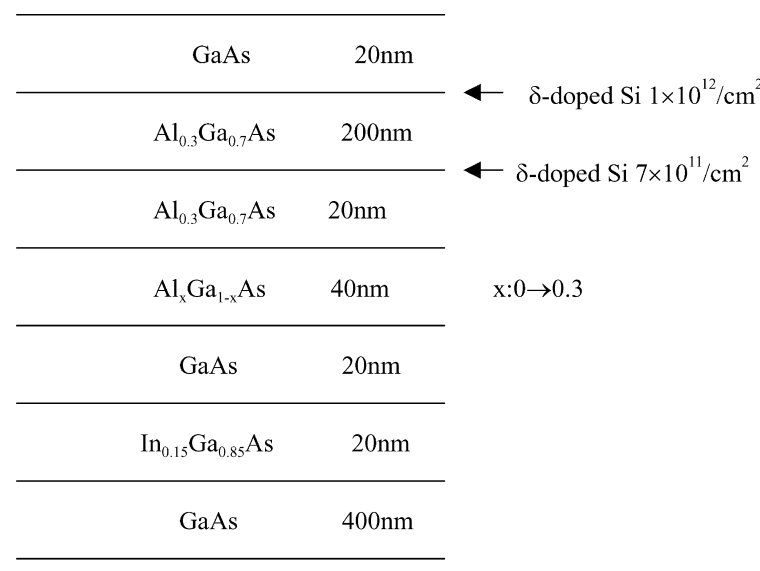

SL buffer GaAs/AlAs $\quad 2 \mathrm{~nm} / 2 \mathrm{~nm} \quad 20$ periods

semi-insulating GaAs substrate

Fig. 1. The sample structure.

contact regions in $\mathrm{N}_{2}$ atmosphere to form ohmic contacts. Magnetotransport measurements were performed with a 0 $15 \mathrm{~T}$ superconductor magnet. We used a top-loading $\mathrm{He}^{3}$ refrigerator for the temperature $T=0.3-1.6 \mathrm{~K}$ and a $\mathrm{He}^{4}$ refrigerator for $T=2.4-4.2 \mathrm{~K}$. The low-frequency AC lock-in technique was used with a current $I=0.1 \mu \mathrm{A}$ to measure the longitudinal and Hall voltages $V_{x x}$ and $V_{x y}$, and we can obtain the longitudinal and Hall resistivities $\rho_{x x}$ and $\rho_{x y}$ by $\rho_{x x}=k V_{x x} / I$ and $\rho_{x y}=V_{x y} / I$, respectively. Here $k=$ $w / l$ with $l$ as the distance between two probes to measure $V_{x x}$ and $w$ as the width of the current channel. From the Shubnikov-de Haas oscillations observed in $\rho_{x x}$ at low $B$, as shown in Fig. 2, the carrier concentration $n=1.3 \times 10^{11} \mathrm{~cm}^{-2}$. The classical mobility $\mu_{\mathrm{c}}=1.0 \times 10^{5} \mathrm{~cm}^{2} / \mathrm{V} \mathrm{s}$ from $\rho_{x x}=1 / n e \mu_{\mathrm{c}}$ at $B=0$. In

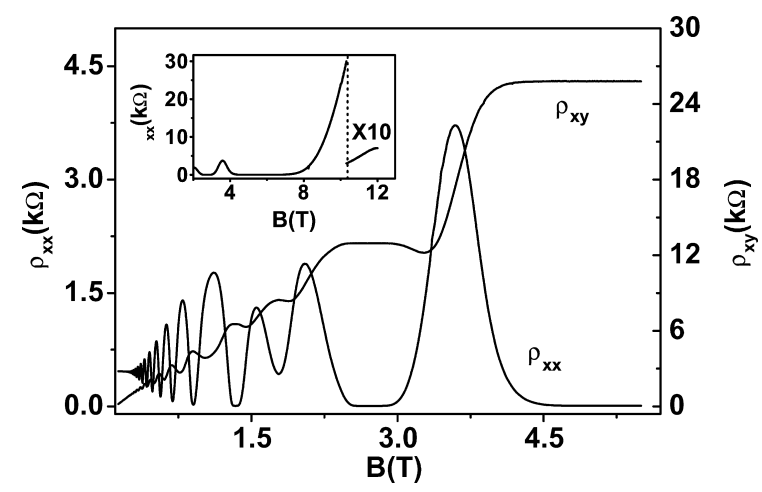

Fig. 2. The curves of the longitudinal and Hall resistivities $\rho_{x x}$ and $\rho_{x y}$ at the temperature $T=0.58 \mathrm{~K}$ when the magnetic field $B=$ $0-5.5 \mathrm{~T}$. At lower $B$ we can observe Shubnikov-de Haas oscillations while at higher $B$ we can observe quantum Hall states of the filling factors $\nu=1$ and 2. The inset shows the curve of $\rho_{x x}$ at $T=0.58 \mathrm{~K}$ when $B=2-12 \mathrm{~T}$. When $B>7 \mathrm{~T}, \rho_{x x}$ increases rapidly and can exceed $h / e^{2} \sim 25.8 \mathrm{k} \Omega$. 
Fig. 2, at high $B$ we can see $\nu=1$ and 2 quantum Hall states in which $\rho_{x x} \rightarrow 0$ and $\rho_{x y} \rightarrow h / \nu e^{2}$. As shown in the inset of Fig. 2, $\rho_{x x}$ increases monotonically as $B$ increases and can exceed $h / e^{2} \sim 25.8 \mathrm{k} \Omega$ when $B>10 \mathrm{~T}$, and the sample enters the insulating state [2]. Since the insulating state and the quantum Hall states of $\nu=1$ and 2 are observed in the $B$ sweep, we can study the $0-1$ and $1-2$ transitions to examine the equivalence between magnetic-field-induced phase transitions in the IQHE.

Transforming $\rho_{x x}$ and $\rho_{x y}$ to the longitudinal and Hall conductivities $\sigma_{x x}=\rho_{x x} /\left(\rho_{x x}^{2}+\rho_{x y}^{2}\right)$ and $\sigma_{x y}=\rho_{x y} /\left(\rho_{x x}^{2}+\right.$ $\left.\rho_{x y}^{2}\right)$, we can plot the temperature-driven flow line diagram $[13,25]$ for the $1-2$ transition in the $\sigma_{x y}-\sigma_{x x}$ plane, as shown in Fig. 3(a). Each solid line corresponds to a temperaturedriven flow line at a magnetic field. At $B=3.4 \mathrm{~T}$ the temperature-driven flow line tends to a fixed point $Q$ marked by the arrow as the temperature $T$ decreases, so the critical magnetic field equals $3.4 \mathrm{~T}$ in the $1-2$ transition $[8,13]$. At the critical magnetic field the filling factor $\nu=1.59$. When
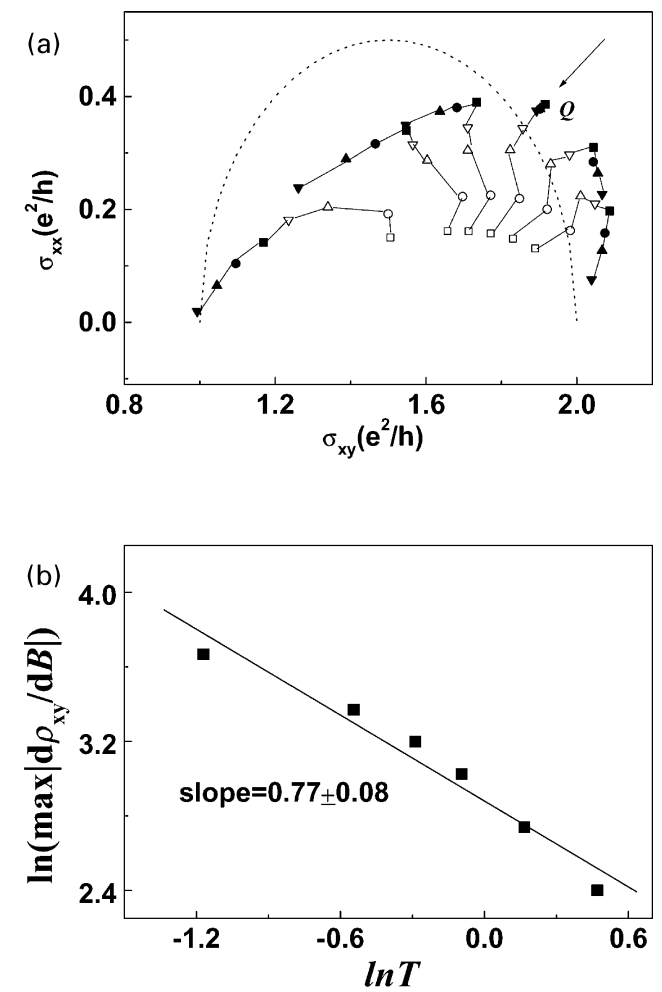

Fig. 3. (a) The temperature-driven flow line diagram for the $1-2 \mathrm{P}-$ $\mathrm{P}$ transition. Each solid line corresponds to a temperature-driven flow line $\left(\sigma_{x y}(T), \sigma_{x x}(T)\right)$ at a magnetic field $B$, and the dot line presents the semicircle $\left(\sigma_{x y}-1.5 e^{2} / h\right)^{2}+\sigma_{x x}^{2}=\left(0.5 e^{2} / h\right)^{2}$. The open squares, circles, up triangles, and down triangles present $\left(\sigma_{x y}, \sigma_{x x}\right)$ at the temperature $T=4,2.4,1.6,1.2 \mathrm{~K}$ and the solid squares, circles, up triangles, and down triangles present $\left(\sigma_{x y}, \sigma_{x x}\right)$ at $T=0.91,0.75,0.58$, and $0.31 \mathrm{~K}$, respectively. At low temperatures, the flow line corresponding to $B=3.4 \mathrm{~T}$ approaches the point $Q=\left(1.9 e^{2} / h, 0.39 e^{2} / h\right)$ marked by the arrow. (b) The line $\ln \left(\max \left|\mathrm{d} \rho_{x y} / \mathrm{d} B\right|\right)-\ln T$ for the $1-2$ transition.
$T>0.91 \mathrm{~K}$, the flow line corresponding to the critical magnetic field deviates from the point $Q$ and hence the critical point is valid only for $T \leq 0.91 \mathrm{~K}$. The point $Q$, however, is located at $\left(1.9 e^{2} / h, 0.39 e^{2} / h\right)$ rather than at the expected point $\left(1.5 e^{2} / h, 0.5 e^{2} / h\right)$ and the universality of critical conductivities is not valid. In Fig. 3(a), in fact, at low temperatures the temperature-driven flow lines do not flow along the semicircle

$\left(\sigma_{x y}-\frac{3}{2} \frac{e^{2}}{h}\right)^{2}+\sigma_{x x}^{2}=\left(\frac{1}{2} \frac{e^{2}}{h}\right)^{2}$

and the semicircle law is not obeyed. Fig. 3(b) shows the curve $\ln \left(\max \left|\mathrm{d} \rho_{x y} / \mathrm{d} B\right|\right)-\ln T$ for the $1-2$ transition when $T=0.31-1.6 \mathrm{~K}$. If the universality of the scaling is valid, $\max \left|\mathrm{d} \rho_{x y} / \mathrm{d} B\right| \propto T^{-\kappa}$ with the critical exponent $\kappa=0.42 \pm$ 0.04 and hence the curve $\ln \left(\max \left|\mathrm{d} \rho_{x y} / \mathrm{d} B\right|\right)-\ln T$ must be a straight line with the slope close to $0.42[5,10]$. However, in Fig. 3(b) the slope of the line $\ln \left(\max \left|\mathrm{d} \rho_{x y} / \mathrm{d} B\right|\right)-\ln T$ is $0.77 \pm 0.08$, deviating from the expected value 0.42 . Hence we do not observe the universal scaling, which could be due to that the temperature is not low enough $[14,15]$ or that the universality of the scaling is broken [10-12]. Therefore, in the 1-2 transition the semicircle law and the universality of the critical conductivities are broken and the expected universal scaling is not observed.

But not all expected properties are broken in the 1-2 transition. In Fig. 3(a) the directions of temperature-driven flow lines are different on the left and right hand sides of the point $Q$, and $Q$ serves as the unique unstable point in the temperature-driven flow line diagram for such a transition as expected $[8,13,25]$. To further study the $1-2$ transition, we can perform the Landau-level addition transformation [1,2]. In a $\mathrm{P}-\mathrm{P}$ transition in the IQHE, such a transformation can be used to subtract the contributions of Landau bands in which all states are filled [2]. Since there is only a filled Landau band in the 1-2 transition, we can define the effective conductivities $\sigma_{x y}^{t}$ and $\sigma_{x x}^{t}$ as

$\sigma_{x y}^{t}=\sigma_{x y}-e^{2} / h$.

$\sigma_{x x}^{t}=\sigma_{x x}$,

to subtract the contribution of this filled Landau band. The corresponding effective longitudinal resistivity [2,14]:

$\rho_{x x}^{t} \equiv \sigma_{x x}^{t} /\left[\left(\sigma_{x x}^{t}\right)^{2}+\left(\sigma_{x y}^{t}\right)^{2}\right]$.

Fig. 4 shows the curves of $\rho_{x x}^{t}$ with respect to $B$ when $T=$ $0.31-0.91 \mathrm{~K}$. Just as $\rho_{x x}$ in the $0-1$ transition, in fact, $\rho_{x x}^{t}$ is temperature-independent at the critical magnetic field $B=$ $3.4 \mathrm{~T}$ [2]. In addition, as $T$ decreases the effective longitudinal resistivity $\rho_{x x}^{t}$ decreases when $B<3.4 \mathrm{~T}$, but increases when $B>3.4 \mathrm{~T}$. So in $\rho_{x x}^{t}$ the critical magnetic field separates two regions with different temperaturedependences. Therefore, the 1-2 transition is mapped to the I-QH transition by the Landau-level addition transformation. Our observations indicate that in the IQHE different magnetic-field-induced transitions can be related by the 


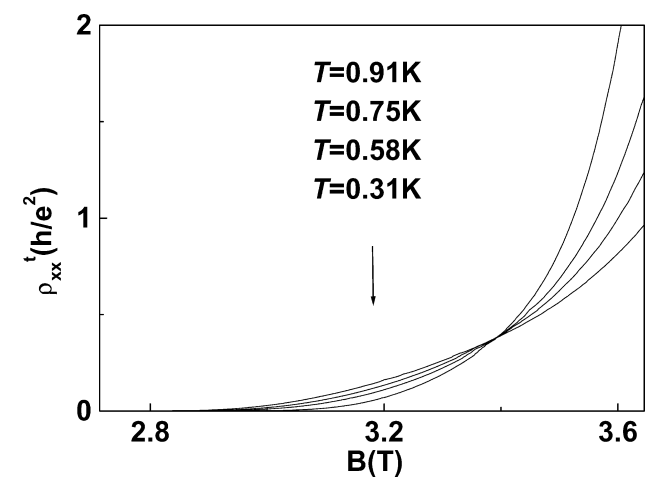

Fig. 4. The curves of effective longitudinal resistivity $\rho_{x x}^{t}(B)$ obtained by performing Landau-level addition transformation on the 1-2 transition at the temperature $T=0.91,0.75,0.58$, $0.31 \mathrm{~K}$. At the critical magnetic field $B=3.4 \mathrm{~T}, \rho_{x x}^{t}$ is $T$ independent.

Landau-level addition transformation even when the semicircle law and the universality of the critical conductivities are broken and the universal scaling is not observed.

We also observed the $0-1$ transition and hence can compare such a transition to the 1-2 transition. Fig. 5 shows the curves of $\rho_{x x}(B)$ at different temperatures near $B=9.8$ T. As $T$ decreases from 0.91 to $0.31 \mathrm{~K}, \rho_{x x}$ increases and the 2DES is in the insulating state when $B>9.8 \mathrm{~T}$, but decreases and the 2DES is in the quantum Hall state of $\nu=1$ when $B<9.8 \mathrm{~T}$. In the same temperature range, $\rho_{x x}$ is temperature-independent at $B=9.8 \mathrm{~T}$ and hence in the $0-1$

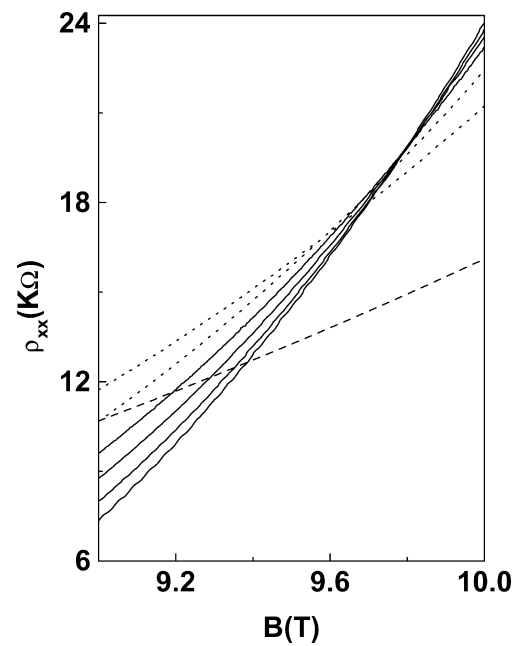

Fig. 5. The traces of the longitudinal resistivity $\rho_{x x}(B)$ at the temperature $T=0.31,0.58,0.75,0.91,1.18,1.6$, and $2.4 \mathrm{~K}$ when the magnetic field $B$ is close to $9.8 \mathrm{~T}$. The solid lines present $\rho_{x x}(B)$ when $T=0.31-0.91 \mathrm{~K}$, and we can see that $\rho_{x x}$ is $T$-independent at $B=9.8 \mathrm{~T}$ in this temperature range. The dotted lines present $\rho_{x x}(B)$ when $T=1.18$ and $1.6 \mathrm{~K}$ and the dashed line corresponds to $T=$ $2.4 \mathrm{~K}$. The longitudinal resistivity $\rho_{x x}$ increases as $T$ decreases at any $B>9.8 \mathrm{~T}$. transition the critical magnetic field is $9.8 \mathrm{~T}$, at which the filling factor $\nu=0.55$. When $T>0.91 \mathrm{~K}$, in Fig. 5 there is no well-defined temperature-independent point and hence the critical point of the $0-1$ transition appears at $0.91 \mathrm{~K}$ as $T$ decreases. At low temperatures, $\rho_{x x}=20 \mathrm{k} \Omega$ while $\rho_{x x}^{t}=10.5 \mathrm{k} \Omega$ at the critical magnetic fields of the $0-1$ and 1-2 transitions, respectively. Therefore, unlike Shahar et al. [2], we cannot see the equivalence between the 1-2 and $0-1$ transitions from the values of $\rho_{x x}^{t}$ and $\rho_{x x}$ at the critical magnetic fields. However, in Figs. 3(a) and 5 both the critical points of the 1-2 and 0-1 transitions appear at $0.91 \mathrm{~K}$ when $T$ decreases, which provides the quantitative evidence for the equivalence between these two transitions in our study.

In conclusion, we have performed magnetotransport measurements on the $2 \mathrm{DES}$ in a $\mathrm{AlGaAs} / \mathrm{InGaAs} / \mathrm{GaAs}$ system. At high fields, the 1-2 P-P and the $0-1 \mathrm{I}-\mathrm{QH}$ transitions are observed, and we can examine the equivalence between magnetic-field-induced phase transitions in the IQHE. In our study, in the 1-2 transition the semicircle law and the universality of critical conductivities are both broken and the universal scaling is not observed. However, not all expected features are broken. In the $\sigma_{x y}-\sigma_{x x}$ plane the directions of the temperature-driven flow lines are unstable near the critical point of the 1-2 transition as expected, and we can still perform the Landau-level addition transformation to map the 1-2 transition to the insulatorquantum Hall conductor transition. The critical points of the 1-2 and 0-1 transitions, in fact, appear at the same temperature as the temperature decreases, which provides the quantitative evidence for the equivalence between these two transitions. Our observations show that the equivalence between magnetic-field-induced phase transitions can be found even when the semicircle law and the universality of critical conductivities are broken and the universal scaling is not observed.

\section{Acknowledgements}

This work is supported by the National Science Council and Ministry of Education of the Republic of China.

\section{References}

[1] S. Kivelson, D.-H. Lee, S.C. Zhang, Phys. Rev. B 46 (1992) 2223.

[2] D. Shahar, D.C. Tsui, M. Shayegan, E. Shimshoni, S.L. Sondhi, Phys. Rev. Lett. 79 (1997) 479.

[3] M. Hilke, D. Shahar, S.H. Song, D.C. Tsui, Y.H. Xie, M. Shayegan, Europhys. Lett. 46 (1999) 775.

[4] A.M.M. Pruisken, Phys. Rev. Lett. 61 (1988) 1297.

[5] H.P. Wei, D.C. Tsui, M.A. Paalanen, A.M.M. Pruisken, Phys. Rev. Lett. 61 (1988) 1294.

[6] A.M. Dykhne, I.M. Ruzin, Phys. Rev. B 50 (1994) 2369. 
[7] M. Hilke, D. Shahar, S.H. Song, D.C. Tsui, Y.H. Xie, D. Monroe, Nature 395 (1998) 675.

[8] B.P. Dolan, Phys. Rev. B 62 (2000) 10278.

[9] Y. Huo, R.E. Hetzel, R.N. Bhatt, Phys. Rev. Lett. 70 (1993) 481.

[10] S. Koch, R.J. Haug, K.v. Klitzing, K. Ploog, Phys. Rev. B 43 (1991) 6828.

[11] D.R. Hang, C.F. Huang, W.K. Hung, Y.H. Chang, J.C. Chen, H.C. Yang, Y.F. Chen, D.K. Shih, T.Y. Chu, H.H. Lin, Semicond. Sci. Technol. 17 (2002) 999.

[12] D. Shahar, M. Hilke, C.C. Li, D.C. Tsui, S.L. Sondhi, J.E. Cunningham, M. Razeghi, Solid State Commun. 107 (1998) 19.

[13] C.-T. Liang, C.F. Huang, Y.-M. Cheng, T.-Y. Huang, Y.H. Chang, Y.F. Chen, Chin. J. Phys. 39 (2001) L305.

[14] R.T.F. van Schaijk, A. de Visser, S.M. Olsthoorn, H.P. Wei, A.M.M. Pruisken, Phys. Rev. Lett. 84 (2000) 1567.

[15] H.P. Wei, S.Y. Lin, D.C. Tsui, A.M.M. Pruisken, Phys. Rev. B 45 (1992) 3926.

[16] T. Ando, J. Phys. Soc. Jpn 55 (1986) 3199.
[17] G. Xiong, S.-D. Wang, Q. Niu, D.-C. Tian, X.R. Wang, Phys. Rev. Lett. 87 (2001) 216802.

[18] P.L. McEuen, A. Szafer, C.A. Richter, B.W. Alphenaar, J.K. Jain, A.D. Stone, R.G. Wheeler, R.N. Sacks, Phys. Rev. Lett. 64 (1990) 2062.

[19] D.N. Sheng, Z.Y. Weng, X.G. Wen, Phys. Rev. B 64 (2001) 165317.

[20] S.-H. Song, D. Shahar, D.C. Tsui, Y.H. Xie, D. Monroe, Phys. Rev. Lett. 78 (1997) 2200.

[21] C.F. Huang, Y.H. Chang, C.H. Lee, H.T. Chou, H.D. Yeh, C.-T. Liang, Y.F. Chen, H.H. Lin, H.H. Cheng, G.J. Hwang, Phys. Rev. B 65 (2002) 045303.

[22] C.H. Lee, Y.H. Chang, Y.W. Suen, H.H. Lin, Phys. Rev. B 58 (1998) 10629.

[23] H.W. Jiang, C.E. Johnson, K.L. Wang, S.T. Hannahs, Phys. Rev. Lett. 71 (1993) 1439.

[24] F. Hohls, U. Zeitler, R.J. Haug, Phys. Rev. Lett. 88 (2002) 036802.

[25] H.P. Wei, D.C. Tsui, A.M.M. Pruisken, Phys. Rev. B 33 (1986) 1488. 